\title{
Quick chip assay using locked nucleic acid modified epithelial cell adhesion molecule and nucleolin aptamers for the capture of circulating tumor cells
}

\author{
Nihal G. Maremanda, ${ }^{1,2, a)}$ Kislay Roy, ${ }^{1, b)}$ Rupinder K. Kanwar, ${ }^{1, c)}$ \\ Vidyarani Shyamsundar, ${ }^{3, \mathrm{~d})}$ Vijayalakshmi Ramshankar, ${ }^{4, e)}$ \\ Arvind Krishnamurthy, ${ }^{5, \mathrm{f}}$ Subramanian Krishnakumar, ${ }^{6,7, \mathrm{~g})}$ and \\ Jagat R. Kanwar ${ }^{1, h)}$ \\ ${ }^{1}$ Nanomedicine-Laboratory of Immunology and Molecular Biomedical Research (NLIMBR), \\ School of Medicine (SoM), Centre for Molecular and Medical Research (C-MMR), Faculty \\ of Health, Deakin University, Geelong, Pigdons Road, Waurn Ponds, Geelong, \\ Victoria 3217, Australia \\ ${ }^{2}$ VIMTA Labs Ltd., \#5, Alexandria Knowledge Park, Genome Valley, Shameerpet, \\ Hyderabad 500078, India \\ ${ }^{3}$ Shree Balaji Dental College, Pallikaranai, Chennai, India \\ ${ }^{4}$ Department of Preventive Oncology, (Research Division) Cancer Institute (WIA), Adyar, \\ Chennai 600 020, India \\ ${ }^{5}$ Department of Surgical Oncology, Cancer Institute (WIA), Adyar, Chennai 600 020, India \\ ${ }^{6}$ Department of Nanobiotechnology, Vision Research Foundation, Kamalnayan Bajaj \\ Institute for Research in Vision and Ophthalmology, Chennai, India \\ ${ }^{7}$ L\&T Ocular Pathology Department, Vision Research Foundation, Kamalnayan Bajaj \\ Institute for Research in Vision and Ophthalmology, Chennai, India
}

(Received 22 June 2015; accepted 1 September 2015; published online 29 September 2015)

The role of circulating tumor cells (CTCs) in disease diagnosis, prognosis, monitoring of the therapeutic efficacy, and clinical decision making is immense and has attracted tremendous focus in the last decade. We designed and fabricated simple, flat channel microfluidic devices polydimethylsiloxane (PDMS based) functionalized with locked nucleic acid (LNA) modified aptamers (targeting epithelial cell adhesion molecule (EpCAM) and nucleolin expression) for quick and efficient capture of CTCs and cancer cells. With optimized flow rates $(10 \mu \mathrm{l} / \mathrm{min})$, it was revealed that the aptamer modified devices offered reusability for up to six times while retaining optimal capture efficiency (>90\%) and specificity. High capture sensitivity (92\%) and specificity $(100 \%)$ was observed in whole blood samples spiked with Caco-2 cells $(10-100$ cells $/ \mathrm{ml})$. Analysis of blood samples obtained from 25 head and neck cancer patients on the EpCAM LNA aptamer functionalized chip revealed that an average count of $5 \pm 3 \mathrm{CTCs} / \mathrm{ml}$ of blood were captured from 22/25 samples (88\%). EpCAM intracellular domain (EpICD) immunohistochemistry on 9 oral squamous cell carcinomas showed the EpICD positivity in the tumor cells, confirming the EpCAM expression in CTCs from head and neck cancers. These microfluidic devices also maintained viability for in vitro culture and characterization. Use of LNA modified aptamers provided added benefits in terms of cost effectiveness due to increased reusability and sustainability of the devices. Our

\footnotetext{
a)nihal.mg@gmail.com

${ }^{\text {b) }}$.roy@deakin.edu.au

c)rupinder.kanwar@deakin.edu.au

d) drvidyaranishyam78@gmail.com

e)r.vijayalakshmi@cancerinstitutewia.in

${ }^{\text {f) }}$ drarvindkrishnamurthy@gmail.com

g)drkrishnakumar_2000@yahoo.com

h) Author to whom correspondence should be addressed. Electronic mail: jagat.kanwar@deakin.edu.au. Tel.: 0061-3-5227-

1148. Fax: 0061-3-5227-3402.
} 
results present a robust, quick, and efficient CTC capture platform with the use of simple PDMS based devices that are easy to fabricate at low cost and have an immense potential in cancer diagnosis, prognosis, and therapeutic planning. (C) 2015 AIP Publishing LLC. [http://dx.doi.org/10.1063/1.4930983]

\section{INTRODUCTION}

Circulating tumor cells (CTCs) can be shed as early as during the formation of the primary tumor. CTCs then get blood-borne and can cause micrometastases that may remain hidden for very long time or even after the thorough surgical removal of the primary tumor. ${ }^{1}$ Detection of CTCs for disease diagnosis, prognosis, and monitoring of the therapeutic efficacy has received increased attention in the recent years. ${ }^{2}$ CTC detection and capture from blood samples of cancer patients is of immense importance in cancer staging, clinical decision making, and also for evaluating the metastatic spread of cancer. ${ }^{3}$ Detection and enumeration of CTCs from peripheral blood non-invasively is referred to as "liquid biopsy." 3,4 Although numerous platforms for CTC capture from blood samples of metastatic cancer patients have been reported, only one of them, namely, CellSearch ${ }^{\circledR}$ technology (a macroscale assay), has been approved by the food and drug administration (FDA). This assay detects the CTCs on basis of multiple receptor expression such as $\mathrm{CD} 45^{-}$, epithelial cell adhesion molecule $(\mathrm{EpCAM})^{+}$, cytokeratin $8^{+}$, cytokeratin $18^{+}$, and cytokeratin $19^{+}$expression in whole blood. However, this assay has shown poor cell capture efficiency. ${ }^{5,6}$ The rarity of occurrence (approximately $1-100 \mathrm{CTCs} / \mathrm{ml}$ of blood) and the high levels of heterogeneity of CTCs are some of the major challenges in developing a CTC-based cancer detection assay with limited available sample. ${ }^{7,8}$ Microfluidics offers a wide variety of applications in developing CTC detection platforms that can be fabricated inexpensively while offering high capture sensitivity and specificity. Several methods have been used for isolation of CTCs based on the physical properties such as shape, size, and deformability; dielectrophoresis, immunospecific surface markers, or magnetic nanoparticle based immunoaffinity. ${ }^{9,10}$

The cell capture probes used in this study are RNA aptamer targeting extracellular domain of EpCAM and DNA aptamer targeting nucleolin protein expression on cancer cells. Nucleolin is essentially a nucleolar non-ribosomal protein that is also expressed in nucleus and cytoplasm and on the cell surface of most cancers. ${ }^{11,12}$ The role of nucleolin in various cellular processes such as DNA transcriptional regulation, pre-RNA processing, transport of rRNA, and cell proliferation has been reported. ${ }^{11}$ Watanabe et al. ${ }^{13}$ recently reported the translocation of intracellular nucleolin to the cell surface as a process occurring during carcinogenesis, as over expression of nucleolin was only seen on the surface of gastric cancer cells while normal gastric epithelial cells showed no expression in the membrane fraction. EpCAM is a transmembrane glycoprotein that was originally identified as a biomarker for colorectal cancers using antibodies in 1979. ${ }^{14}$ Although EpCAM is expressed by most normal epithelial cells, ${ }^{15}$ reports have also shown the over expression of EpCAM in several carcinomas. ${ }^{16,17}$ Expression of EpCAM has also been reported in cancer stem cells, which are a small group of tumor cells that show self-renewability and tumor initiating properties. ${ }^{18,19}$ Recent reports have also shown that controlled proteolysis of the intracellular domain of EpCAM (EpICD) causes its translocation to the nucleus, which ultimately aids in cell proliferation. ${ }^{20}$ Hence, these markers are optimum for the capture of cancer cells and CTCs. However, the assay requires highly specific probes that can bind to the target with high affinity.

Aptamers as detection probes for cell surface markers have shown true potential for molecular recognition and targeting. Specific aptamers can be synthesized for any cell type or targeting specific cell surface markers to capture cells in microfluidic devices. ${ }^{21}$ Aptamers are single stranded DNA, RNA, or modified oligonucleotide sequences that show target specificity and recognition to various molecules ranging from proteins, cell surface antigens, biomolecules such as ATP and also cells and cell membranes ${ }^{22,23}$ by forming unique secondary and tertiary structures. ${ }^{24}$ Generation of aptamers is carried out through an in vitro selection procedure called systemic evolution of ligands by exponential enrichment (SELEX), which involves the selection 
of specific aptamers from a large library of random DNA or RNA molecules on competitive binding with target molecules followed by purification and amplification. ${ }^{3}$ Use of flat channel devices for immobilizing sgc8, TD05, and Sgd5 aptamers (DNA aptamers) for multiplexed capture of various leukemia cell lines with high specificity was reported by $\mathrm{Xu}$ et al. ${ }^{25}$ Sheng et $a{ }^{26}{ }^{26}$ reported the development of a device with micropillars coated with KDED2a-3 and KCHA10 aptamers (DNA aptamers) to specifically isolate DLD-1 and HCT 116 cells (colorectal cancer cells) spiked in whole blood.

One of the most important requirements for aptamers as diagnostic or therapeutic tools is adequate stability in in vivo conditions or in biological fluids. As any oligonucleotide, aptamers are easily degraded by nucleases. Modifications with locked nucleic acid (LNA) has been the most commonly used method for increasing stability of aptamers. LNA are ribonucleotides consisting of bicyclic high affinity analogues, which mimic RNA conformation by introducing a methylene bridge that connects the $2^{\prime}$-oxygen of ribose with the $4^{\prime}$-carbon. Upon hybridization of DNA/RNA with LNA, there is a rise in the melting temperature $\left(\mathrm{T}_{\mathrm{m}}\right)$ of the duplex. ${ }^{27,28}$ LNA modified aptamers are known to exhibit increased thermal stability, specificity to targets, high cellular uptake, and increased half-life in blood. ${ }^{27-30}$

We proposed that incorporation of LNA in the aptamers would aid in developing a platform that is robust and allows reusability without the need for modifying the microchannel surfaces after each run. Several groups across the globe have reported label free CTC capture devices, which are based on the physical properties of the cells such as shape, size, and deformability; and dielectrophoretic signatures of cell membranes. ${ }^{31}$ A continuous-flow system was developed by Shim et al. ${ }^{32}$ for rapid processing of blood samples and isolation of tumor cells with increased purity based on the dielectrophoretic potential of cells. Size based isolation of rare cells from blood samples has also been reported using multi-stage multi-orifice flow fractionation $^{33}$ and a double spiral microchannel ${ }^{34}$ that solely depend on the hydrodynamic forces within the flow channels for cell separation. These methods have emerged as potential alternatives to the more commonly used immunospecific surface markers or magnetic nanoparticle based immunoaffinity methods. However, these methods involve complex and expensive fabrication procedures, various sample fractionation steps, and infrastructure for controlled flow conditions and electric field generation. Thus, the microfluidic devices used in this study consisted of flat microchannels branching out from a single inlet into four capture microchannels (chip 1) or capture microchambers (chip 2). The efficiency of these chips was evaluated without the need for engineering complex 3-dimensional structures within the channels for improving interaction between cells under flow conditions and modified channel surfaces.

This is the first study to use simple microfluidic devices functionalized with LNA modified aptamers targeting EpCAM and nucleolin markers for quick and efficient capture of CTCs. Moreover, we came up with a well-designed, easy to manufacture, and cost effective microfluidic device that can specifically and efficiently capture rare cells and can be re-used multiple times with high sensitivity and specificity (Figures 1(a) and 1(b)). A schematic representation of aptamer immobilization and translocation of EpICD is shown in Figures 1(c) and 1(d). We evaluated the diagnostic applicability of the EpCAM LNA aptamer functionalized microfluidic devices using blood samples of head and neck cancer patients.

\section{RESULTS AND DISCUSSION}

\section{High serum stability of LNA modified aptamers}

To study the stability of LNA modified aptamers in biofluids, LNA modified aptamers were suspended in phosphate buffer saline (PBS) containing 10\% fetal bovine serum (FBS) for time periods ranging from 0 to 5 days and were run on $1 \%$ agarose gel. It was observed that both EpCAM and nucleolin LNA aptamers were highly stable in $10 \%$ serum. Nucleolin LNA aptamer was stable for up to 5 days; however, EpCAM LNA aptamer showed slight degradation after $72 \mathrm{~h}$ (Figure 2(a)). It is possible that the nucleolin LNA aptamer was more stable than the EpCAM LNA aptamer because the EpCAM aptamer is a RNA aptamer, while the nucleolin aptamer is a DNA aptamer. Previously published studies have reported that DNA 

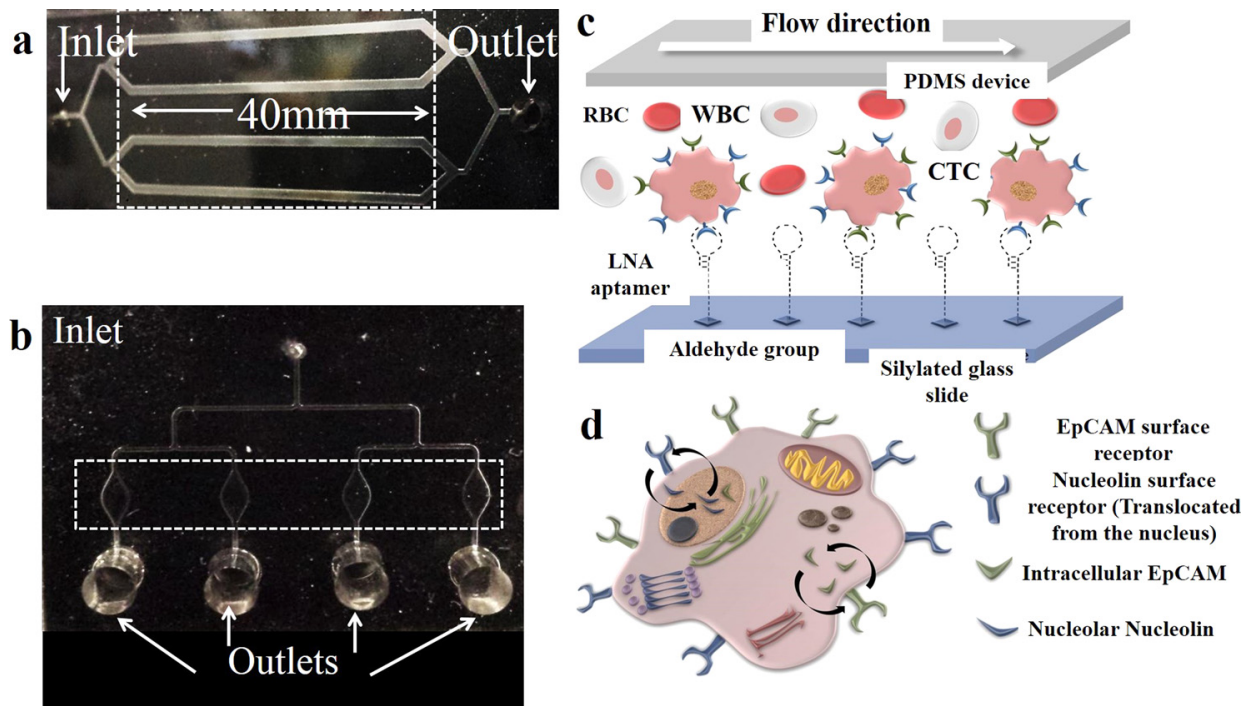

FIG. 1. Schematic of the microfluidic devices designed for cell capture studies: (a) Represents chip 1, a flat channel device consisting of four $40 \mathrm{~mm}$ long cell capture microchannels that are $1 \mathrm{~mm}$ wide and $100 \mu \mathrm{m}$ deep. All the channels are connected at the inlet $(0.75 \mu \mathrm{m}$ diameter) and converge into a single outlet reservoir (diameter- $3 \mathrm{~mm})$. (b) is representative of the second microfluidic device, chip 2 that consists of four elliptical cell capture chambers that are $3 \mathrm{~mm}$ long, $1.5 \mathrm{~mm}$ wide (at the center), and $100 \mu \mathrm{m}$ deep. Each cell capture chamber leads to a separate outlet reservoir (diameter-3 mm). The dotted areas represent the cell capture channels/chambers. (c) A schematic representation of LNA aptamer immobilization on silylated glass slides and specific cell capture. (d) Schematic representing the intracellular translocation of EpCAM and nucleolin surface receptors on cancer cells.

aptamers are comparatively more stable than RNA aptamers due to the existence of structural heterogeneity within the RNA, various nucleotide modifications, and an increased susceptibility to form 3D structures. ${ }^{35,36}$ These results suggest that the LNA aptamers can be used to develop robust cell capture platforms without the need for stringent storage and transportation conditions.

\section{Successful immobilization of LNA modified aptamers on silylated glass slides}

To evaluate the efficiency of aptamer immobilization on silylated glass slides, surface roughness of aptamer coated slides was analyzed with atomic force microscopy (AFM). Freshly coated silylated slides with $20 \mu \mathrm{M}$ EpCAM LNA aptamer solution was used to analyze surface roughness in comparison with uncoated slides. A two fold increase in surface roughness (yellow area) upon aptamer immobilization was observed, suggesting the successful functionalization of silylated surfaces with aptamers (Figure 2(b)). These results suggest that the method used for aptamer immobilization via Schiff base formation allowed successful binding of aptamers on the silylated glass slides (Figure 2(c)). Formation of a Schiff base between the aromatic amines (on the nucleotide bases of the aptamer) and aldehyde or carbonyl group (present on the silylated glass slides) through nucleophilic addition is the basis for aptamer immobilization. ${ }^{37}$

\section{Specific capture of cancer cells on LNA aptamer functionalized surfaces}

The purpose for designing chip 2 with separate outlets for each capture chamber (unlike chip 1) besides its applicability for cell capture was to study its use for characterization of the captured cells. The presence of separate outlets allows cells in each chamber to be characterized for a separate marker/protein. This enables multiple staining of the captured cells on a single device simultaneously (see Figure S1 in the supplementary material in Ref. 38).

The performance of the microfluidic devices functionalized with LNA modified aptamers targeting EpCAM and nucleolin was first evaluated using Caco-2 cells (human colorectal adenocarcinoma cells). Serum free medium spiked with cancer cells at a concentration of 
$\mathbf{a}$

Time (Hours)

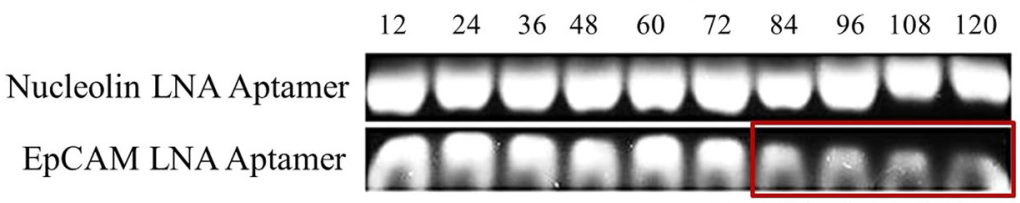

Possible degradation
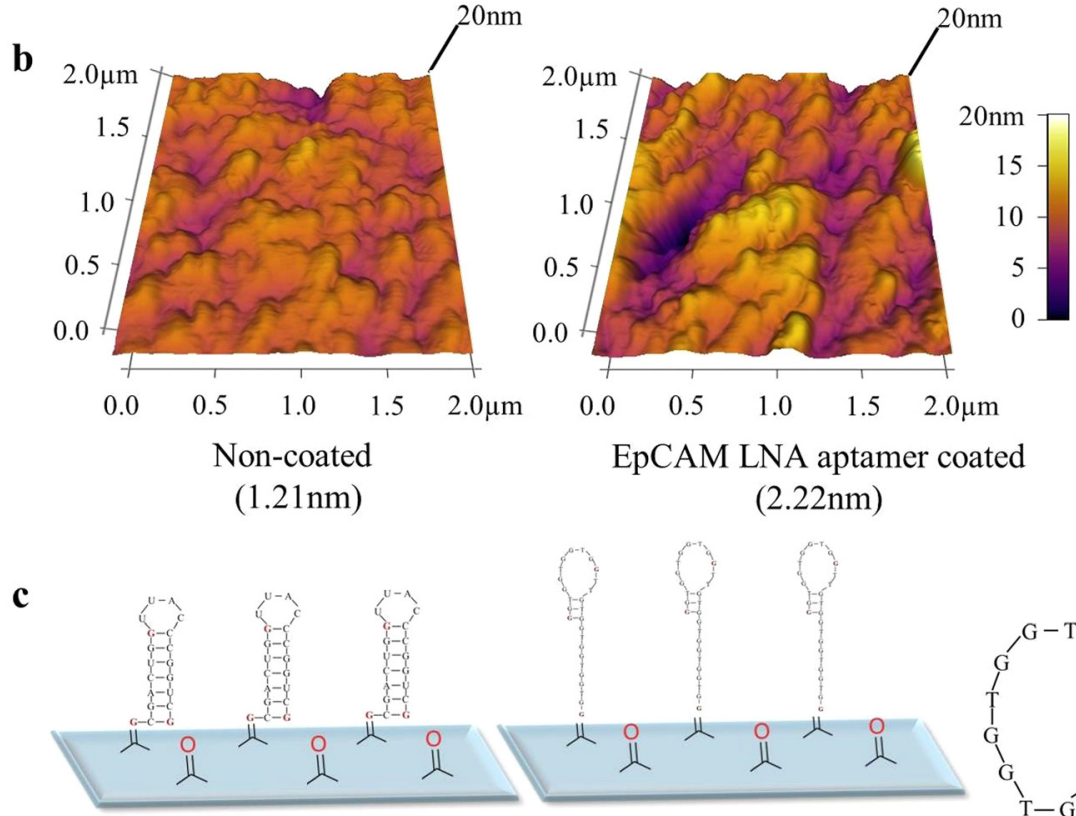

LNA aptamers coated silylated glass slide

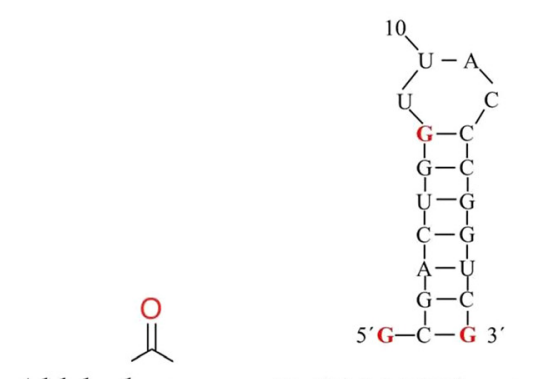

Aldehyde groups EpCAM LNA aptamer

(M Fold)

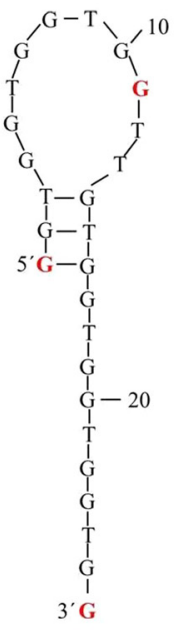

\section{Nucleolin LNA aptamer (M Fold)}

FIG. 2. Aptamer functionalized microfluidic devices: (a) Representative agarose gel images showing high serum stability of LNA modified aptamers in PBS containing $10 \%$ fetal bovine serum from 12 to $120 \mathrm{~h}$. (b) Representative images showing increase in surface roughness upon aptamer immobilization on silylated glass slides. (c) Schematic representation of aptamer immobilization on silylated glass slides. Formation of a Schiff base between the aromatic amines (on the nucleotide bases of the aptamer) and a carbonyl group (present on the silylated glass slides) through nucleophilic addition is the basis for aptamer immobilization. $\mathrm{M}$ fold (using the $\mathrm{M}$ fold computational tool) structures are predictive of the aptamer folding and hybridization (the $\mathrm{M}$ fold for the aptamers were determined in a previous publication from our laboratory ${ }^{64}$ ).

1000 cells/ml was flowed through the microfluidic devices at a flow rate of $10 \mu \mathrm{l} / \mathrm{min}$ to evaluate the efficiency of the aptamer enabled cell capture (Figure 3(a)). However, the image data and the corresponding numerical data cannot be absolutely correlated as the images shown here are only representative data obtained for a specific area of the device from a single micro-channel/chamber. For instance, in case of chip 1, each microchannel is $40 \mathrm{~mm}$ long and it is not possible to show images of the entire area. Hence, the area with the highest captured cells 

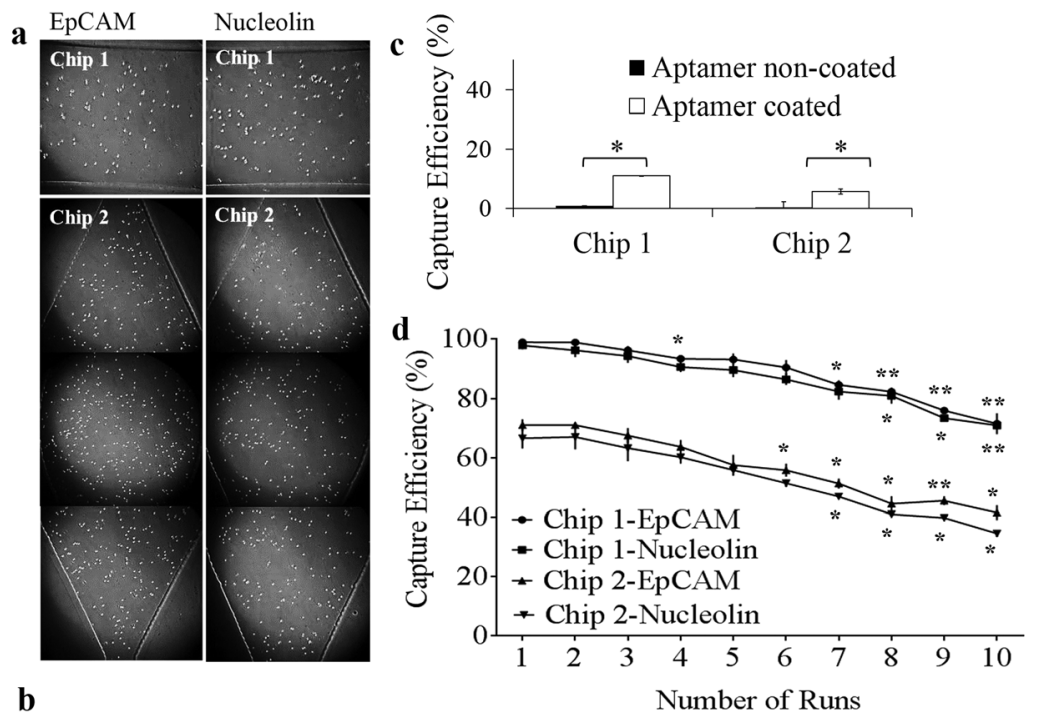

b

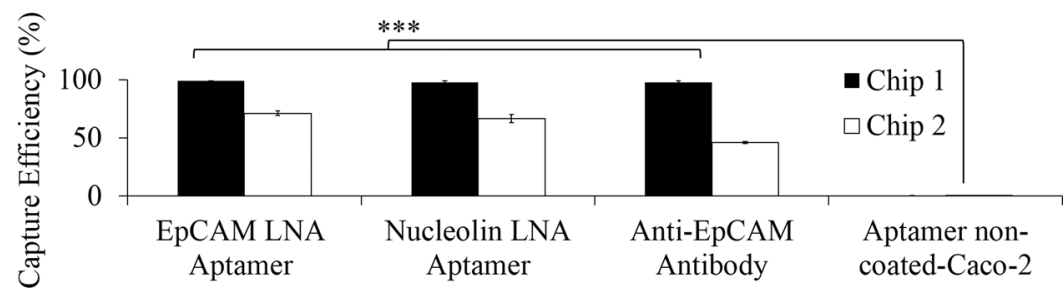

FIG. 3. Cell capture efficiency on aptamer functionalized microfluidic devices: (a) Representative bright field images of Caco-2 cells captured on EpCAM and nucleolin LNA aptamer functionalized surfaces of chips 1 and 2. (b) Comparison of capture efficiency of Caco-2 cells on aptamer and antibody modified surfaces. (c) Evaluation of capture specificity using FHs 74 Int cells on EpCAM LNA aptamer coated and non-coated surfaces. The results are represented as mean \pm SEM obtained from three repeats, $* * * \mathrm{p}<0.001$. (d) Capture yield of 1000 Caco- 2 cells flowed through EpCAM and nucleolin LNA aptamer functionalized chip 1 and chip 2 microchannels after each run for ten repeats. The results are represented as mean \pm SEM obtained from three technical repeats, ${ }^{* * *} \mathrm{p} \leq 0.001, * * \mathrm{p} \leq 0.01, * \mathrm{p} \leq 0.05$.

(imaging was done at the same area for all the channels in all the experiments performed) has been shown for all experiments. Thus, these percentages will not correlate with the cell numbers seen in the images as only a certain portion of the microchannels can be represented in the images.

A capture efficiency as high as $99 \%$ and $98 \%$ was obtained on EpCAM LNA aptamer and nucleolin LNA aptamer functionalized surfaces of chip 1. However, chip 2 showed a capture efficiency of $71 \%$ and $66.5 \%$ on EpCAM LNA aptamer and nucleolin LNA aptamer functionalized surfaces (Figure 3(b)). The capture efficiency was calculated based on the total number of unbound cells collected at the outlets. All the cells collected at the outlets from the start of the experiment (introduction of cell suspensions) till the final wash step (with PBS) were counted using a haemocytometer to determine capture efficiency. Cells were counted after centrifuging the cell suspensions collected at the outlet to pellet the cells, which were immediately resuspended into fresh medium before being counted on a haemocytometer.

The decreased capture efficiency of chip 2 can be attributed to the decreased surface area of chip. The increased surface area of each microchannel in chip $1(40 \mathrm{~mm} \times 1 \mathrm{~mm})$ allows enhanced immobilization of aptamers ultimately aiding in efficient cell capture, when compared to that of microchambers of chip $2(3 \mathrm{~mm} \times 1.5 \mathrm{~mm})$. To confirm the specificity of cell capture, Caco-2 cells were also flowed through microfluidic devices without aptamer modification. Similarly, FHs 74 Int cells (normal colon epithelial cells) were also flowed through devices with and without aptamer modification (Figure 3(c)). It was seen that neither Caco-2 nor FHs 74 Int cells were captured on surfaces without aptamer modification. The use of scrambled oligonucleotides as control was eliminated as previous studies reported non-specific capture of 
target and control cells on surfaces immobilized with random oligonucleotides. ${ }^{39}$ A small percentage of FHs 74 Int cells were captured (11\% on chip 1 and $5.7 \%$ on chip 2) on EpCAM LNA aptamer modified surfaces, which can be expected because of the epithelial origin of the cells. ${ }^{40,41}$ This assay was mainly done to test the ability of the microfluidic chip to differentiate between the cancer cells and the normal cells.

Both the EpCAM and nucleolin aptamers used in this study have been reported to have exceptional cancer targeting properties and have been used extensively for targeted therapy. ${ }^{42-46}$ However, it can be said that the cancer cell viability is not affected during the cell capture assays, as there are no unbound aptamers available in the microchannels. Those present are immobilized via Schiff base formation on silylated glass surfaces, hence eliminating the possibility of the aptamer internalization into the cancer cells. The continuous flow conditions as well as the short time periods of interaction between the flowing cancer cells and the immobilized aptamers eliminate any possible anti-cancer effects of the aptamers on the cancer cells. The factors suggest that the use of these aptamers does not affect cell viability, capture sensitivity, or specificity.

\section{Multiple reusability of aptamer functionalized devices for cancer cell capture}

Microfluidics has been used extensively for capture and isolation of CTCs commonly relying on affinity based capture. Many of these cell capture platforms have shown efficient capture of rare cells. ${ }^{5,47}$ However, only a few reports ${ }^{48,49}$ have shown the ability of these devices to allow subsequent release of captured cells while maintaining them in viable conditions for further studies. A recent study by Zhu et al. ${ }^{49}$ reported the use of sgc8c aptamer (DNA aptamers targeting protein tyrosine kinase 7) modified microfluidic devices for efficient capture of CCRF-CEM (acute lymphoblastic leukemia cells) and subsequent temperature mediated release of captured cells while maintaining their viability. A similar study employed the use of antiPSMA (prostate-specific membrane antigen) aptamers (RNA aptamers) coated microfluidic devices to efficiently capture prostate cancer cells and later release them using trypsin. ${ }^{48}$ It would also be highly beneficial if the devices can be readily reused after releasing bound cells for running more samples without the loss of cell capture function. To address this, LNA modified aptamers targeting EpCAM and nucleolin were immobilized on silylated glass slides separately to validate the reusability of the modified microfluidic devices. Caco- 2 cells spiked in serum free medium at a concentration of 1000 cells $/ \mathrm{ml}$ were introduced into the aptamer modified devices (at a flow rate of $10 \mu \mathrm{l} / \mathrm{min}$ ) repeatedly to evaluate the capture efficiency after each run. Trypsin-mediated release of captured cells was performed after each run (for a total of 10 runs) to remove all bound cells on-chip. To eliminate any inconsistencies or variations between devices, the experiments were performed in two different devices. With optimized flow rates obtained from earlier studies $(10 \mu \mathrm{l} / \mathrm{min})$, it was revealed that the EpCAM LNA aptamer modified chip 1 devices offered reusability for up to six times while maintaining the capture efficiency above 90\%, whereas the nucleolin LNA aptamer modified devices showed similar capture efficiency for up to four repeats. Although the purification of aptamers is an expensive procedure and LNA modification of bases only increases the expenditure, the stability and shelf life of LNA aptamers and the multiple reusability of chip by a single patient at subsequent time intervals justify the cost-effectiveness of the device. It is, however, important to note that both aptamers showed $>80 \%$ capture yield for up to eight repeats on chip 1 . These results show the regenerative ability of LNA modified aptamer functionalized device for repeated cell capture. The arbitrary cut-off of six times was determined based on the capture efficiency. All repeats showing capture efficiency $>90 \%$ were considered significant. The capture efficiency fell below $90 \%$ from the seventh repeat. Besides this, LNA modification also increases clinical and bio-analytical applicability of aptamers due to factors such as increased stability, resistance to high temperatures, ease of synthesis, ease of introducing modifications/ functional groups for increasing stability, reduced immunogenicity and toxicity, and the ability to generate large volumes of highly specific aptamers to a wide range of target molecules. ${ }^{39,50,51}$ Chip 2 also showed a similar trend in cell capture function after repeated runs. 
Although lower than that of chip 1, chip 2 showed a higher capture yield (for up to six runs) compared to that on anti-EpCAM antibody immobilized devices (Figure 3(d)).

\section{High capture sensitivity on aptamer functionalized devices}

The rarity of occurrence of CTCs in peripheral blood samples of cancer patients poses a major challenge in developing a sensitive cell capture system. ${ }^{7,8}$ To simulate this in vivo condition, Caco-2 cells were spiked in serum free medium at concentrations ranging from 10 to 1000 cells $/ \mathrm{ml}$ and were flowed through aptamer functionalized devices to evaluate the capture sensitivity. Chip 1 functionalized with LNA modified aptamers showed high capture sensitivity even with low cell numbers. The EpCAM LNA aptamer modified chip 1 device was able to capture up to $8 / 10$ cells $(80 \%)$ that were flowed through, while the same device functionalized with nucleolin LNA aptamer captured $7 / 10$ cells $(70 \%)$. Similar results were also obtained when chip 1 was flowed with cell suspensions containing 100 cells (Figures 4(a) and 4(b)). Both LNA modified aptamer functionalized devices showed $>90 \%$ capture efficiency for cell suspensions containing 100 cells. These results were better than the standard-aptamer based cell capture techniques. ${ }^{5}$ Although published reports suggest overexpression of nucleolin in colon cancer $^{52}$ and other cancers, ${ }^{53}$ expression levels of surface nucleolin in comparison with EpCAM have not been studied well. Therefore, it can only be postulated that Caco-2 cells express higher levels of EpCAM in comparison to surface nucleolin based on the capture yield with these aptamers. However, aptamer modified chip 2 showed efficient capture of only 2-3 cells when flowed with cell suspensions containing 10 cells. Approximately 50\% reduction in capture yield was seen on chip 2 with cell suspensions containing 100 cells compared to 1000 cells.

\section{Efficient capture of cancer cells spiked in whole blood on aptamer modified surfaces}

To simulate the ability to capture rare CTCs in peripheral blood of cancer patients, predetermined concentrations of Caco-2 cells (1000 cells) were spiked in whole blood samples obtained from BALB/C nude mice. It is important to evaluate the sensitivity and specificity of a cell capture device to capture target cells present in small numbers in a heterogeneous cell suspension. It was seen that chip 1 showed 92\% cell capture efficiency with EpCAM LNA aptamers, while nucleolin LNA aptamer functionalized chip 1 showed capture of $88 \%$ of Caco2 cells from blood (Figures 4(c) and 4(d)). These results were slightly better than the state of art techniques (90\% capture efficiency with $84 \%$ purity) for CTC capture from blood. ${ }^{52}$ Although a small percentage of nonspecific binding was observed when whole blood spiked with cancer cells was flowed through, the subsequent PBS washes removed most of the non-specifically bound cells, thereby increasing capture specificity values. A study reported by Shen et al., 5 employing the use of gold nanoparticles immobilized with $\operatorname{sgc} 8$ aptamers on microchannel surfaces, also reported a fall in capture efficiency when cancer cells were spiked in whole blood at predetermined concentrations. However, this device showed a much lower sensitivity in capture yield $(60 \%)$ when compared to the results obtained in the present study with chip $1 .^{5}$ Chip 2 showed a capture efficiency of $65 \%$ and $61 \%$ with EpCAM and nucleolin LNA aptamers, respectively. The results showed a slight decrease in capture efficiency of cancer cells spiked in whole blood compared to that in medium. This decrease in capture yield can be attributed to the presence of a vast number of haemocytes that temporarily block the interaction of the immobilized aptamers with the target cells in the direction of their flow. It was, however, evident that the aptamer modified devices specifically captured only cancer cells. Chip 1 modified with EpCAM LNA aptamers showed a 7\% fall in capture efficiency, while the nucleolin LNA aptamer modified devices showed a $10 \%$ fall when compared with cell capture in medium. Aptamer modified chip 2 devices showed approximately $6 \%$ fall in capture efficiency of cancer cells in blood compared to that in medium. These results showed the ability of the aptamer functionalized devices to capture target cells specifically from a vast background of bystander cells (Figure 4(d)). To further illustrate the clinical applicability of this cell capture method, whole blood was spiked with 10,50 , and 100 Caco-2 cells to mimic the rarity of 

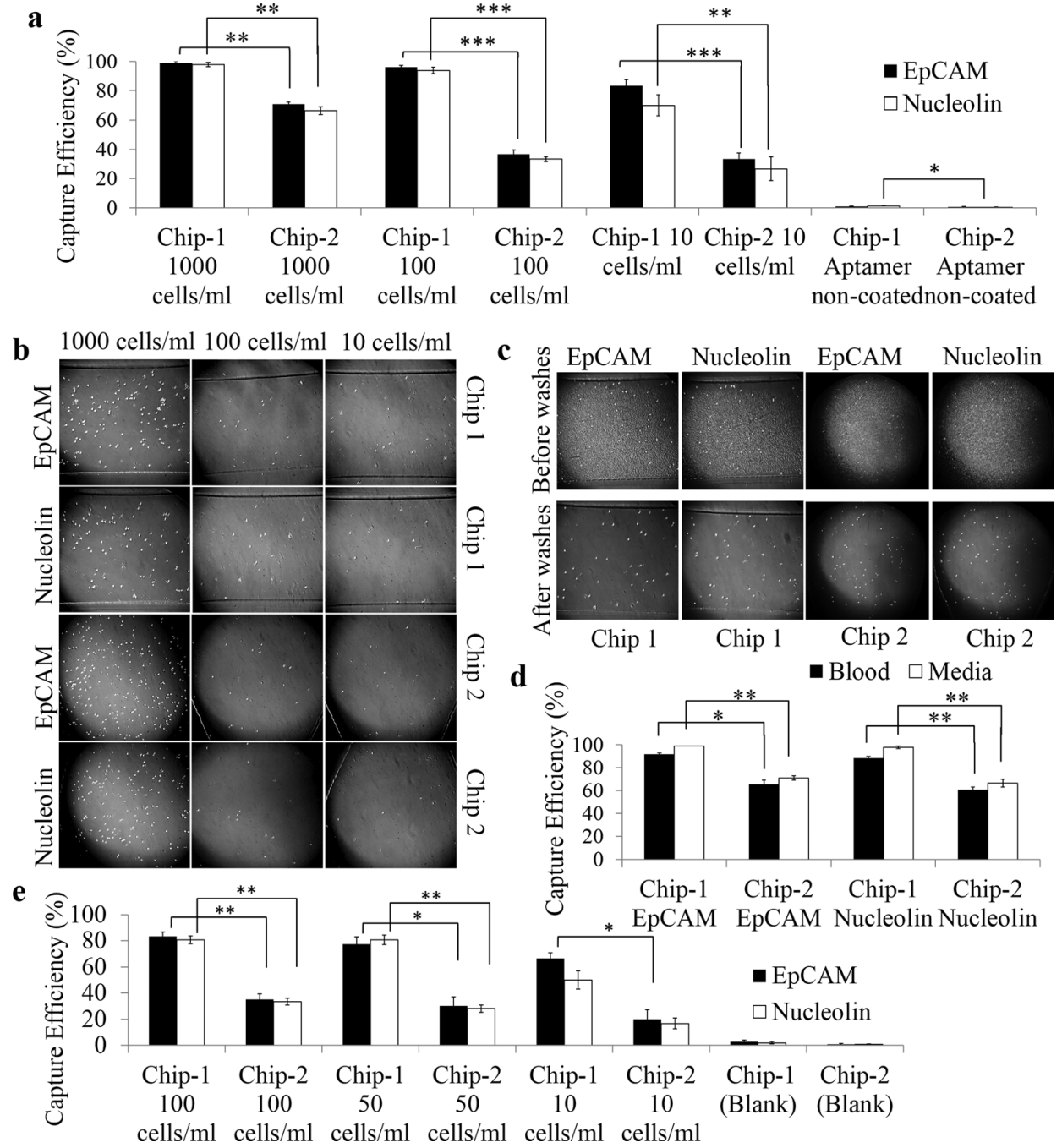

FIG. 4. Cell capture sensitivity from medium and blood samples: (a) Comparative analysis of capture sensitivity of Caco-2 cells (spiked in low concentrations) on aptamer functionalized surfaces. The results are represented as mean \pm SEM obtained from three repeats, $* * * \mathrm{p} \leq 0.001$. (b) Representative bright field images of Caco-2 cells (spiked in low concentrations) captured on microchannels of chip 1 functionalized with EpCAM LNA aptamer and nucleolin LNA aptamer. (c) Representative bright field images of Caco- 2 cells captured on microchannels of chips 1 and 2 functionalized with EpCAM and nucleolin LNA aptamer. Capture specificity was evaluated for 1000 Caco-2 cells spiked in whole blood samples. (d) Capture yield of 1000 Caco-2 cells spiked in whole blood in comparison to that in medium. Error bars represent standard deviation obtained from three repeats. (e) Aptamer enabled capture of Caco-2 cells spiked in whole blood at low concentrations on chips 1 and 2 . The results are represented as mean \pm SEM obtained from three repeats, *** $\mathrm{p} \leq 0.001$, $* * \mathrm{p} \leq 0.01, * \mathrm{p} \leq 0.05$.

occurrence of CTCs in blood samples of cancer patients. EpCAM LNA aptamer modified chip 1 captured 6/10 Caco-2 cells that were flowed through the microchannels, while chip 2 effectively captured 2/10 cells. Similar results were seen with nucleolin LNA aptamer modified devices. Capture efficiency of $77 \%$ and $80 \%$ was observed with chip 1 functionalized with EpCAM and nucleolin aptamers, respectively, when flowed with 50 Caco-2 cells per ml sample. Both aptamers showed greater than $80 \%$ cell capture on chip 1 flowed with blood samples spiked with 100 cells (Figure 4(e)).

\section{Capture of various cancer cells based on EpCAM/nucleolin/stem cell marker expression}

To determine the capture yield based on EpCAM and nucleolin expression levels in different cancer cell lines, chip 1 functionalized with aptamers was used to flow through cancer cells 
mixed with equal proportions of methylene blue stained non-cancerous cells. The $\%$ expression was calculated based on total number of cells captured. Therefore, the \% expression is equal to the cell capture efficiency. The capture efficiency was in turn calculated based on the total number of unbound cells collected at the outlets. All the cells collected at the outlets from the start of the experiment (introduction of cell suspensions) till the final wash step (with PBS) were counted using a haemocytometer to determine capture efficiency (Table I). Cell capture based on surface marker expression was performed with (i) DU145 cells (human prostate cancer cells) mixed with RWPE-1 cells (human prostate epithelial cells); (ii) MCF-7 cells (human breast cancer cells) mixed with RWPE-1 cells; (iii) HepG2 cells mixed with FHs 74 Int cells; and (iv) Caco-2 cells mixed with FHs74 Int cells (Figure 5(a)). Capture efficiency varied with each cell line based on surface EpCAM/Nucleolin expression on these cell lines. ${ }^{16,53-55}$ The normal cells were pre-stained with $1 \%$ methylene blue before mixing with un-stained Caco-2 cells to visualize non-specific on-chip capture of cells in real time. It was observed that DU145 and MCF-7 cells showed higher EpCAM expression (>99\%) compared to Caco-2 and HepG2 cells, whereas HepG2 showed the highest nucleolin expression (>98\%) compared to other cancer cells (Figure 5(b)). The capture purity on EpCAM LNA aptamer functionalized devices was $<90 \%$ with all four cancer cell lines as both the non-cancer cell lines (FHs 74 Int and RWPE-1) that were used show low level expression of EpCAM. ${ }^{41}$ A cell capture device that can capture cells specifically based on surface marker expression can be used to stratify patient populations based on the expression levels of various markers. This will aid appropriate treatment planning and disease management. Cancer cells showing stem cell like properties were also flowed through aptamer modified surfaces of chip 1 to evaluate the capture efficiency (Table II). Cancer cells (DU145, MCF-7, Caco-2) were first immuno-magnetically sorted based on cell surface stem cell marker (EpCAM, CD44, and CD133) expression. These cancer stem cells were mixed with equal proportions (1:1) of methylene blue stained normal cells (RWPE-1 and FHs74 Int cells) before introducing into aptamer modified chip 1. Greater than 98\% cell capture was seen with cancer stem cells on both EpCAM and nucleolin aptamer modified devices (Figure 5(c)). The capture purity fell on EpCAM aptamer modified devices as all epithelial cells express EpCAM on their cell surface, which resulted in capture of a few FHs 74 Int and RWPE-1 cells. It was observed that the capture efficiency of cancer stem-like cells was higher than that of unsorted cancer cells. These results demonstrate the ability of the chip for capture of both cancer cells and cancer stem-like cells without lowering their viability (Figure 5(d)). This is the first study to show efficient capture of cancer stem-like cells on LNA-aptamer functionalized microchannel surfaces. The isolation and characterization of rarely occurring CTCs showing stemness is of enormous benefit to understand various processes such as drug

TABLE I. Percentage EpCAM and nucleolin expression in various cancer cell lines.

\begin{tabular}{lcccc}
\hline \hline & \multicolumn{2}{c}{$\%$ Cells expressing EpCAM } & & \\
\cline { 2 - 3 } Cell lines & Cancer cells & Normal cells & Efficiency \% & Purity \% \\
\hline Caco-2 : FHs 74 Int & 97 & 12 & 97 & 88 \\
DU145 : RWPE-1 & 99.46 & 11.57 & 99.46 & 88.43 \\
MCF-7 : RWPE-1 & 99.34 & 13.34 & 99.34 & 86.66 \\
HepG2 : FHs 74 Int & 97.98 & 10.69 & 97.98 & 89.31 \\
& & & \\
Cell lines & $\%$ Cells expressing nucleolin & & \\
\hline Caco-2 : FHs 74 Int & Cancer cells & Normal cells & Efficiency \% & Purity \% \\
DU145 : RWPE-1 & 95.78 & 5.67 & 95.78 & 94.33 \\
MCF-7 : RWPE-1 & 98.00 & 3.56 & 98 & 96.44 \\
HepG2 : FHs 74 Int & 97.23 & 4.78 & 97.23 & 95.22 \\
\hline \hline
\end{tabular}



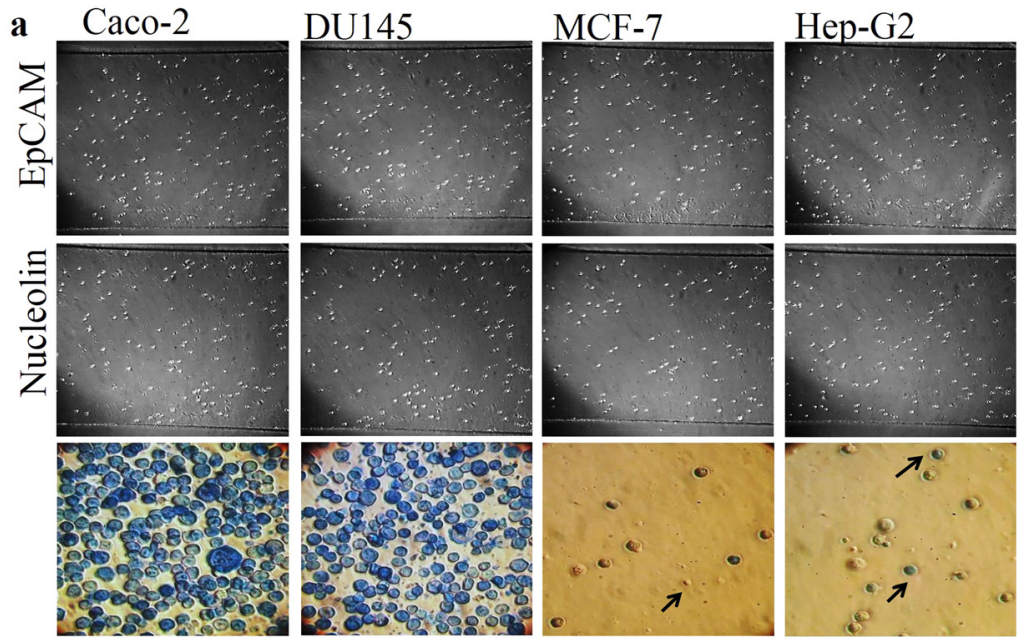

MB stained FHs

MB stained

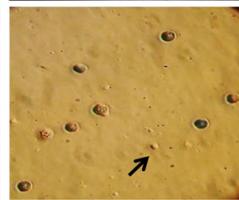

74 Int cells

RWPE-1 cells

Captured FHs Captured

74 Int cells

RWPE-1 cells
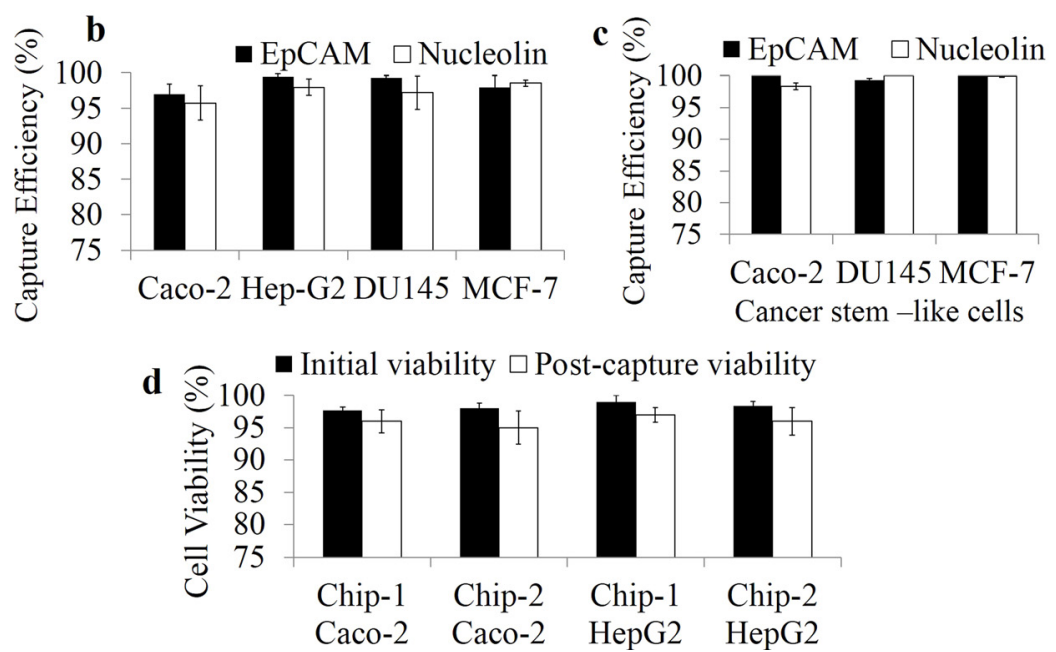

FIG. 5. Capture efficiency of various cell lines and cancer stem-like cells on aptamer functionalized devices: (a) Representative bright field images of various cancer cells captured on EpCAM LNA aptamer (upper panel) and nucleolin LNA aptamer (middle panel) functionalized microchannels of chip 1. Arrowheads represent captured FHs 74 Int and RWPE-1 cells (blue) along with cancer cells. (b) Cancer cell capture based on EpCAM/nucleolin expression on aptamer functionalized chip 1. (c) Aptamer enabled capture of cancer stem-like cells on chip 1. Percentage EpCAM and nucleolin expression in various cell lines and cancer stem-like cells was estimated based on capture efficiency. Error bars represent standard deviation obtained from three repeats. (d) Cell viability post-capture on EpCAM LNA aptamer functionalized devices. Error bars represent standard deviation obtained from three repeats.

resistance, metastasis, and tumor recurrence. ${ }^{16}$ This strategy of capturing rare cells with LNA modified aptamers shows a huge potential for understanding cancer related processes to develop novel therapeutic strategies.

\section{Efficient capture of CTCs from whole blood on EpCAM LNA aptamer functionalized devices}

Ultimately, to illustrate the clinical applicability of the LNA aptamer functionalized microfluidic devices to isolate CTCs with high sensitivity and specificity, whole blood samples obtained from head and neck cancer patients were run on chip 1 immobilized with EpCAM LNA aptamer. Blood samples obtained from patients with pre-cancerous lesions (such as erythroplakia or leukoplakia) were used as negative controls. Freshly obtained blood samples from patients with advanced stage head and neck cancer $(n=25)$ and pre-cancerous lesions $(n=8)$ 
TABLE II. Percentage EpCAM and nucleolin expression in various cancer stem-like cells.

\begin{tabular}{|c|c|c|c|c|}
\hline \multirow[b]{2}{*}{ Cancer stem-like cells } & \multicolumn{2}{|c|}{$\%$ EpCAM expression $^{\mathrm{a}}$} & \multirow[b]{2}{*}{ Efficiency $\%$} & \multirow[b]{2}{*}{ Purity $\%$} \\
\hline & Cancer cells & Normal cells & & \\
\hline $\mathrm{Caco} 2\left(\mathrm{EpCAM}^{+}, \mathrm{CD}_{133^{+}}\right)$and FHs $74 \mathrm{Int}$ & 100.00 & 14.35 & 95.78 & 85.65 \\
\hline DU145 $\left(\mathrm{EpCAM}^{+}, \mathrm{CD} 44^{+}\right)$and RWPE-1 & 98.25 & 9.58 & 98 & 88.67 \\
\hline \multirow[t]{2}{*}{ MCF7 $\left(\mathrm{EpCAM}^{+}, \mathrm{CD}_{133^{+}}\right)$and RWPE-1 } & 99.34 & 12 & 97.23 & 87.34 \\
\hline & \multicolumn{2}{|c|}{$\%$ Nucleolin expression $^{\mathrm{a}}$} & & \\
\hline Cancer stem-like cells & Cancer cells & Normal cells & Efficiency $\%$ & Purity $\%$ \\
\hline Caco2 $\left(\mathrm{EpCAM}^{+}, \mathrm{CD}_{133^{+}}\right)$and $\mathrm{FHs} 74 \mathrm{Int}$ & 98.34 & 4.35 & 95.78 & 93.99 \\
\hline DU145 $\left(\mathrm{EpCAM}^{+}, \mathrm{CD}^{4} 4^{+}\right)$and RWPE-1 & 98.78 & 2.29 & 98 & 96.49 \\
\hline MCF7 $\left(\mathrm{EpCAM}^{+}, \mathrm{CD} 133^{+}\right)$and RWPE-1 & 100.00 & 3.50 & 97.23 & 96.5 \\
\hline
\end{tabular}

${ }^{a}$ Percentage EpCAM and nucleolin expression in various cancer cells was estimated based on capture efficiency. Capture purity was deduced based on the total number of methylene blue stained normal cells captured on chip.

were processed on chip 1 immobilized with EpCAM LNA aptamer (Table III and Figure 6(a)). Only EpCAM LNA aptamer functionalized devices were used with blood samples obtained from head and neck cancer patients. This was done as available literature suggests the overexpression of EpCAM in such cancers. ${ }^{56}$

The authenticity of the captured cells to be CTCs (not haematocytes) was confirmed using confocal microscopy that evaluated the specificity of cell capture. Various parameters of the CTC capture were analysed using the formulas (provided in the subsection "CTC capture and characterization from blood samples" in the "Methods" section), and it was determined that the device showed very high diagnostic efficiency and moderate sensitivity and specificity (Table IV). CTCs captured on-chip were identified as nucleated (DAPI stained) EpCAM positive cells (FITC stained) (Figure 6(b)), which is in accordance with previous reports. ${ }^{16,56}$ According to

TABLE III. Clinical information of samples used for CTC quantification.

\begin{tabular}{lc}
\hline \hline Characteristics & Sample No. \\
\hline 1. Gender & 28 \\
Male & 5 \\
Female & \\
2. Age group & 5 \\
25-35 & 14 \\
36-50 & 12 \\
51-65 & 2 \\
66-80 & \\
3. Postoperative stage & 6 \\
III & 19 \\
IV & 8 \\
Pre-cancerous lesions & \\
4. Tumor location & 17 \\
Buccal mucosa & 8 \\
Tongue & 3 \\
Gingiva & 3 \\
Hard palate & 1 \\
Floor of mouth & 1 \\
Mandibular alveolus & \\
\hline \hline
\end{tabular}


$\mathbf{a}$
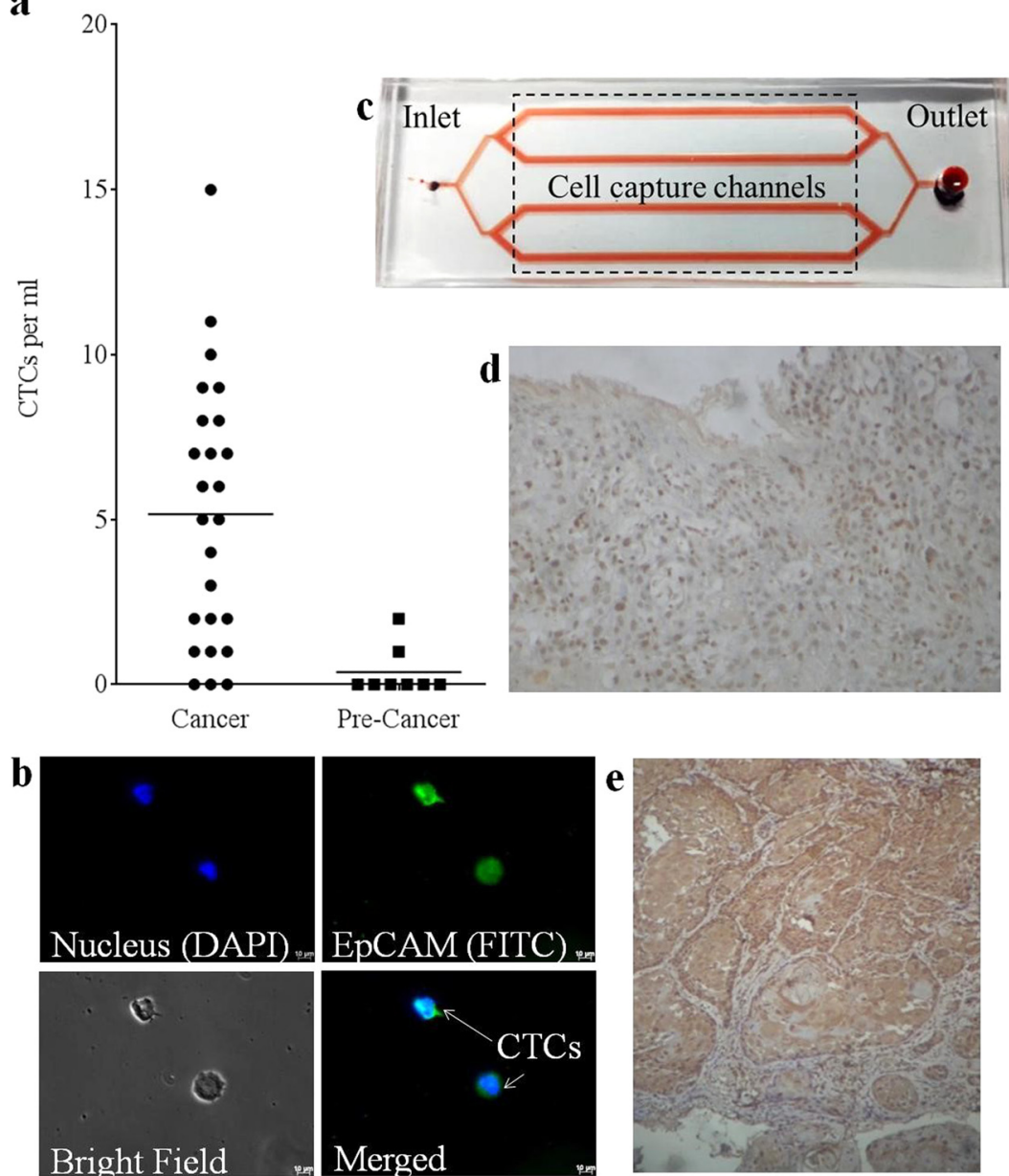

FIG. 6. CTC capture and characterization from blood samples of head and neck cancer patients: (a) Quantification of CTCs captured from blood samples of patients with cancer and pre-cancerous lesions. (b) Representative fluorescence microscopy images of CTCs captured from blood sample of head and neck cancer patients. (c) Layout of the microfluidic device immobilized with EpCAM LNA aptamer used for capture of CTCs from cancer samples. Photomicrograph showing the cytoplasmic and nuclear localization of EpCAM ICD in (d) gingival squamous cell carcinoma (20× magnification) and (e) tongue squamous cell carcinoma $(10 \times$ magnification).

the results obtained, 22/25 blood samples from advanced stage head and neck cancer patients showed the presence of CTCs. Each sample was run thrice, and the average of the three repeats (for each sample) has been shown. 19 samples showed $\geq 2 \mathrm{CTCs} / \mathrm{ml}$ of blood analyzed (Figure $6(\mathrm{c})$ ). An average of $5 \pm 3 \mathrm{CTCs} / \mathrm{ml}$ of blood was identified in the cancer samples that were analyzed. Similar results $(5 \pm 4 \mathrm{CTCs} / \mathrm{ml})$ have been reported by Yoon et al. from blood samples of lung, pancreatic, and breast cancer patients. The study used a polydimethylsiloxane (PDMS) based device having a silicon substrate with patterned gold structures (flower shaped) on which graphene oxide sheets-functionalized with EpCAM antibodies were adsorbed.$^{57}$ However, blood

TABLE IV. Diagnostic evaluation of the CTC capture using patient samples.

\begin{tabular}{lcc}
\hline \hline S. No & Diagnostic evaluation criterion & $\%$ value \\
\hline 1 & Sensitivity & 89 \\
2 & Specificity & 75 \\
3 & Diagnostic efficiency & 85 \\
4 & Predictive positive & 92 \\
5 & Predictive negative & 73 \\
\hline \hline
\end{tabular}


samples obtained from patients with pre-cancerous lesions showed zero to negligible number of CTCs. Immunohistochemistry (Figures 6(d) and 6(e)) for EpICD was performed on oral squamous cell carcinoma tissue sections to confirm the expression of EpCAM in tumor cells. The immunohistochemistry results showed both cytoplasmic and nuclear staining in all the samples $(n=9)$ studied. The intensity of the cytoplasmic and nuclear staining varied among the tumors studied, with majority of the tumor cells showing 70\%-80\% positivity (the cytoplasmic and nuclear expression of EpCAM in tumor samples). The percentage of the EpICD positive cells in the tumor are presented in Table V. These results further suggest the role of EpICD in cancer progression on nuclear translocation.

These results demonstrated the specificity and sensitivity of LNA aptamer modified devices and their potential applicability in a clinical setting to determine therapeutic efficacy or chances of disease recurrence based on CTC number in a patient. These devices can also be used in combination with other aptamers to target cell-specific markers for developing screening assays for various cancers. This is the first study to report the use of LNA-aptamers to capture CTCs from cancer samples on simple, flat channel microfluidic devices without the need for complicated 3D structures. These microfluidic devices are not only easy to manufacture but also offer a more robust platform for CTC capture with regenerative functionality without the need for stringent storage and transportation conditions.

\section{Conclusions}

The results obtained in this study showed that LNA modified aptamer based simple microfluidic devices as CTC capture platforms hold immense potential in cancer detection, monitoring, and management. Advantages of LNA modified aptamers such as high serum stability and strong covalent binding with modified glass surfaces allow the development of a robust CTC capture platform. These features also extend the applicability of these flat channel microfluidic devices for analyzing multiple samples on a single device functionalized with LNA modified aptamers. Device reusability offers wide applicability in sample processing and detection in rural or remote areas without the need for extensive laboratory reagents and equipment. Sensitive and specific capture of rare cancer cells from whole blood samples offered by LNA aptamer functionalized devices on simple flat channel devices further improves their clinical applicability in disease detection at early stages and therapeutic monitoring. High sensitivity in detecting rare cells also allows detection and characterization of cancer cells showing stem cell properties that are a minority among the other CTCs. This information can be used in planning appropriate treatment strategies and move towards personalized treatments. They can also be put to use for detecting minimal residual disease (MRD) for leukaemia and for solid tumors. The presence of a small number of cancer cells in circulation post treatment is a major cause of cancer relapse and metastasis. ${ }^{56,57}$ These aptamer modified microfluidic devices can be used to accurately detect MRD for monitoring treatment response and also as a predictive tool for cancer recurrence. The simple design, the low cost for the fabrication of these devices, and the ease of

TABLE V. Clinicopathological information of EpCAM ICD on the oral squamous cell carcinoma tissue sections.

\begin{tabular}{lccccc}
\hline \hline S. No & Age/Sex & Clinicopathological information & Grade and clinical stage & Treatment & EpCAM ICD \% \\
\hline 1 & 50 & Cheek ulcer of 17 months duration & Grade 2, T4aN1 & Chemoradiation & 70 \\
2 & 70 & Gingival growth 1 month duration & Grade 3, T4a N1M0 & Chemoradiation & 80 \\
3 & $46 / \mathrm{M}$ & Cheek ulcer of 3 months duration & Grade 2-3, T3N0 & Chemoradiation & 60 \\
4 & 32/M & Buccal mucosa growth for 2 months & Grade 3, T4aN2b & Chemoradiation & 80 \\
5 & $41 / \mathrm{F}$ & Cheek ulcerative growth for 3 months & Grade 2-3, T4aN2c & Chemoradiation & 60 \\
6 & $65 / \mathrm{M}$ & Cheek ulcer for 2 months & Grade 1-2, T4aN1 & Chemoradiation & 70 \\
7 & $42 / \mathrm{M}$ & Sulcus growth for 3 months & Grade 2, T3nN1 & Chemoradiation & 70 \\
8 & $57 / \mathrm{M}$ & Hard palate growth for 6 months & Grade 2-3, T4aN2b & Chemoradiation & 70 \\
9 & $62 / \mathrm{M}$ & Oropharynx painful ulcer for 2 months & Grade 3, T4aN1 & Chemoradiation & 60 \\
\hline \hline
\end{tabular}


modification with aptamers targeting various cancer specific markers enable their use as important diagnostic tools for a wide range of cancers.

\section{METHODS}

\section{Device fabrication}

The microfluidic chips used in this study were comprised of PDMS devices reversibly bonded on to microscope glass slides. Fabrication of all PDMS devices was performed at Melbourne Centre for Nanofabrication, Melbourne, Victoria. Commercially available silylated glass slides were used. Standard lithography and deep reactive-ion etching (DRIE) etching methods were used for PDMS device fabrication. DRIE etching was the method used for creating the design on the wafers with specific dimensions that was later used for fabricating the PDMS devices. The PDMS device fabrication was performed prior to them being bounded onto the silylated glass slides. Therefore, the etching process does not affect the glass surfaces. Briefly, silicon wafers (diameter: $4 \mathrm{in}$. and thickness: $500 \mu \mathrm{m}$ ) were dipped in piranha solution (30\% $\mathrm{H}_{2} \mathrm{O}_{2}$ and $\mathrm{H}_{2} \mathrm{SO}_{4}$ ) for 15 min followed by rinsing with deionized water for $10 \mathrm{~min}$ and blow drying with nitrogen. The silicon wafers were first coated with hexamethyldisilazane (HMDS) to enhance photoresist adhesion by spinning at $3000 \mathrm{rpm}$ for $30 \mathrm{~s}$ and then allowed to rest for a minute at room temperature. This was followed by spin coating with AZ4652 photoresist with a $4 \mu \mathrm{m}$ thick layer at $3000 \mathrm{rpm}$ for $30 \mathrm{~s}$. The wafers were then soft baked at $100^{\circ} \mathrm{C}$ for a minute and then allowed to re-hydrate at room temperature for $15 \mathrm{~min}$. They were then exposed using a contact mask aligner at $180 \mathrm{~mJ} / \mathrm{cm}^{2}$, developed in 4:1 (Water: AZ400K) developer for one minute and rinsed with deionized water. Pre-cleaning was performed with de-scum (a pre-cleaning step to remove any residual photo resist from trenches and microchannels on the wafers for maintaining uniformity on the surfaces) in DRIE tool for $10 \mathrm{~s}$. Bosch processing to etch target depth of $100 \mu \mathrm{m}$ was carried out. Visual inspection and depth verification were performed via stylus profiler. Silicon master wafers were stripped in oxygen plasma and re-cleaned with piranha as above. Master molds were activated with oxygen plasma for $1 \mathrm{~min}$ at 500 mTorr in a Harrick plasma unit. Molds were immediately transferred to a vacuum desiccator with $20 \mu \mathrm{l}$ (tridecafluoro-1,1,2,2-tetrahydrooctyl)-1-trichlorosilane and brought to vacuum (300-500 mTorr) for $1 \mathrm{~h}$. Silanized molds were then transferred to a casting dish, where 10:1 PDMS (Sylgard 184) was poured to a depth of approximately $0.75-1 \mathrm{~cm}$. Casting tray was degassed for $1 \mathrm{~h}$ under vacuum before being transferred to a surface leveled oven at $80^{\circ} \mathrm{C}$ for $2 \mathrm{~h}$. Devices were extracted from master mold by manual dicing with a straight edge and scalpel.

\section{Surface modification with LNA modified aptamers}

$20 \mu \mathrm{M}$ LNA modified aptamers were suspended in $3 \times$ Standard saline citrate (SSC) ( $0.15 \mathrm{M}$ sodium chloride, $15 \mathrm{mM}$ sodium citrate, $\mathrm{pH}: 7)$ and pumped onto silylated microscope slides mounted with the PDMS chip. Formation of a Schiff base between the aromatic amines (on the nucleotide bases of the aptamer) and a carbonyl group (present on the silylated glass slides) through nucleophilic addition is the basis for aptamer immobilization. Incubation with the aptamer solution was done for $4 \mathrm{~h}$ in a humidified chamber to enable rehydration. The channels were then cleaned with $0.2 \%$ SDS for a minute followed by washing with nuclease free water twice for a minute each. Washing with sodium borohydride solution $(10 \mathrm{mg}$ of $\mathrm{NaBH}_{4}$ dissolved in $3 \mathrm{ml}$ of PBS and $1 \mathrm{ml}$ of ethanol; PBS was prepared in nuclease free water) was performed for $5 \mathrm{~min}$. The slides were submerged in water for $2 \mathrm{~min}$ at $95^{\circ} \mathrm{C}$ then rapidly washed with $0.2 \%$ SDS for a minute. The slides were rinsed well in water twice and air dried before storing them in dark at room temperature until further use. ${ }^{58}$ The aptamers used in this study are nucleolin DNA aptamer: 5'-GGTGGTGGTGGTTGTGGTGGTGGTGG-3' and EpCAM RNA aptamer: 5'-GCGACUGGUUACCCGGUCG-3'. The regions in the aptamer sequence with the LNA modification are given in bold. The LNA modified aptamers were procured from Ribotask, Denmark, and have been well characterized earlier. ${ }^{59}$ 


\section{Evaluation of surface roughness with AFM}

An Asylum Research Cypher AFM was used, in tapping mode, to study the surface roughness of the aptamer coated surfaces. Silylated glass slides were tested for an increase in surface roughness after aptamer functionalization as compared to that of non-coated slides. Argyle Light, Asylum Research, Santa Barbara, CA, was used for data analysis.

\section{Cell culture}

The cell lines used in this study, namely, human colon adenocarcinoma cells (Caco-2), human hepatocellular carcinoma cells (HepG2), human mammary adenocarcinoma cells (MCF7), human prostate carcinoma cells (DU145), human intestinal epithelial cells (FHs 74 Int), and human prostate epithelial cells (RWPE-1), were obtained from American type culture collection (ATCC), Manassas, VA, USA. Eagle's Minimum Essential Medium (EMEM) was used for the culture of Caco-2, MCF7, HepG2, and DU145; Hybri-Care medium for FHs 74 Int cells and keratinocyte serum free medium supplemented with epidermal growth factor for RWPE-1 cells was obtained from Gibco, Australia. The medium for the other cell lines was supplemented with $10 \%$ fetal bovine serum purchased from Bovogen, Australia, and antimycotic-antibiotic or penicillin/streptomycin purchased from Gibco, Australia. Media changes were done every 3 days and the cells were subcultured with $0.25 \%$ Trypsin/EDTA (obtained from Gibco, Australia) upon reaching a confluency of $80 \%-90 \%$.

\section{Serum stability analysis of LNA modified aptamer}

The EpCAM and nucleolin LNA aptamers were suspended in nuclease free water and stored at $-20^{\circ} \mathrm{C}$ as stock solutions. The serum stability of each of these aptamers was determined by incubating $20 \mu \mathrm{M}$ aptamer solutions with PBS containing $10 \%$ fetal bovine serum for 0 to $120 \mathrm{~h}$. These solutions were then run on $1 \%$ agarose gel at $100 \mathrm{~V}$ for $35 \mathrm{~min}$ and visualized with SYBR safe on Bio-Rad gel doc imaging system.

\section{Cell capture assays}

Immediately before performing the cell capture experiments, the cells were trypsinized and resuspended in fresh serum free medium. Cell viability was evaluated prior to running the experiment with trypan blue exclusion assay. The cells were mixed with an equal volume of trypan blue stain and counted on haemocytometer for evaluating cell viability. Cell suspensions showing $>95 \%$ cell viability were used for subsequent analysis. The cells were diluted to the required concentration in serum free medium before introducing into the microfluidic devices. Aptamer immobilized slides were blocked with $3 \%$ bovine serum albumin (BSA) and $0.1 \%$ Tween-20 (prepared in nuclease-free water) for $1 \mathrm{~h}$. Channels were washed with PBS (prepared in nuclease-free water) thrice. Cell suspensions with predetermined concentrations of cells (approximately $300 \mu \mathrm{l}$ ) were injected at a flow rate of $10 \mu \mathrm{l}$ per minute. Medium at the outlet reservoir was collected regularly in $1.5 \mathrm{ml}$ tubes for counting total number of unbound cells. The channels were washed with PBS (prepared in nucleasefree water) to remove unbound cells and medium. The cell suspensions were introduced into the devices using a syringe pump (PHD Ultra 4400 series, Harvard apparatus) connected at the inlet of the devices with a $1 \mathrm{ml}$ syringe via polymer tubing. The total number of cells captured was determined by counting the number of unbound cells collected at the outlet reservoirs

$$
\% \text { Capture efficiency }=\frac{\text { Number of target cells captured }}{\text { Total number of target cells introduced }} \times 100
$$

$$
\% \text { Capture purity }=\frac{\text { Number of target cells captured }}{\text { Number of target cells captured }+ \text { number of control cells captured }} \times 100 \text {. }
$$




\section{Evaluation of reusability of LNA aptamer functionalized devices}

Trypsin (approximately $150 \mu \mathrm{l}$ ) at $37^{\circ} \mathrm{C}$ was injected at a flow rate of $20 \mu \mathrm{l} / \mathrm{min}$ to release bound cells. The process to remove all bound cells typically took around 3 to $5 \mathrm{~min}$. Channels were washed with PBS (prepared in nuclease-free water) to remove any traces of trypsin. Once the channels were free of all bound cells and trypsin, fresh cell suspensions (showing $>95 \%$ cell viability) were flowed through to determine the capture yield for each run. This process was repeated for a total of 10 times, and the capture yield was determined after each run based on the total number of unbound cells collected at the outlet reservoir. The experiments were discontinued if the cell viability fell below 95\%. The Chip-bound aptamer coated slides were washed with PBS, drained of any liquid, and stored in dark at room temperature. The same slides were reused on availability of fresh cells the following day.

\section{Cancer cell capture from whole blood}

Blood from nude mice (balb/c nu nu) collected in an EDTA vacutainers was spiked with predetermined concentration of Caco-2 cells to evaluate the capture specificity. The whole blood samples were used within $2 \mathrm{~h}$ of collection. To test the specificity of the cell capture method, $1 \mathrm{ml}$ whole blood was spiked with 1000 Caco-2 cells and flowed through EpCAM LNA aptamer and nucleolin LNA aptamer modified devices at a flow rate of $10 \mu \mathrm{l} / \mathrm{min}$. Capture yield was determined after washing with PBS at a flow rate of $15 \mu \mathrm{l} / \mathrm{min}$.

\section{Cell staining with methylene blue}

Cells were stained with $1 \%$ methylene blue solution to enable real time evaluation of capture specificity. Freshly trypsinized cells were incubated for $15 \mathrm{~min}$ at $37^{\circ} \mathrm{C}$ in $1 \%$ methylene blue solution prepared in serum free medium. The stained cells were pelleted by centrifugation at $1500 \mathrm{rpm}$ for $5 \mathrm{~min}$. The pellet was rinsed in PBS thrice before resuspending in serum free medium. Methylene blue stained cells were then mixed with unstained cancer cells at predetermined concentrations to evaluate capture specificity in real time. The cell mixture containing target cells (unstained cancer cells) and control cells (stained non-cancerous cells) were flowed through the devices at flow rates ranging from 5 to $30 \mu \mathrm{l} / \mathrm{min}$ to determine the optimal flow conditions for obtaining high capture efficiency with a high capture purity.

\section{Sorting of cancer cells based on cancer stem cell marker expression}

Freshly trypsinized cancer cells were flowed through sorting columns contained magnetic beads specific to CD133, CD44, and EpCAM. These columns were obtained from MACS Milteny Biotec. Magnetic separation on the MACS column was performed using anti-biotin magnetic beads to separate cells labelled with biotin-conjugated antibodies against the above mentioned markers. The sorted cells were cultured for $24 \mathrm{~h}$ before performing cell capture experiments.

\section{Cell recovery and viability post-capture}

All experiments were performed using freshly trypsinized cells with $>95 \%$ cell viability (using trypan blue exclusion assay). After the cell suspensions were flowed through the microfluidic devices, the captured cells were released using $0.25 \%$ trypsin-EDTA at $37^{\circ} \mathrm{C}$ flowed at $20 \mu \mathrm{l} / \mathrm{min}$ and collected at the outlet reservoirs. The released cells were stained with trypan blue and counted on haemocytometer to determine cell viability.

\section{Blood sample collection}

Blood samples from patients with advanced head and neck cancers $(n=25)$ and oral premalignant lesions $(\mathrm{n}=8)$ were collected from Head and Oncology clinic at Cancer Institute (WIA), Chennai, Tamil Nadu, India, and Balaji Dental College and Hospital, Chennai, Tamil Nadu, India. 
Informed consent was obtained from each of participating patient as per the Institutional ethical guidelines (IRB No:SBDCECM 106/14/30). The blood samples were collected in EDTA vacutainers and were processed within $4 \mathrm{~h}$ of sample collection. The staging was done based on TNM (tumor, nodal, metastatic) classification given by International agency for research on cancer (IARC) screening group, World health organization. ${ }^{59,60}$ Details of the samples collected are enlisted below.

\section{СTC capture and characterization from blood samples}

Freshly collected blood samples were diluted in equal proportion with PBS before being introduced into the microfluidic device chip 1, functionalized with EpCAM LNA aptamer. Cell capture assays were performed as discussed above. The captured cells were analyzed for EpCAM expression and for the presence of a nucleus using immunofluorescence. This involved fixing the captured cells with $4 \%$ paraformaldehyde (PF) solution for $20 \mathrm{~min}$ at room temperature. The channels were then washed with PBS thrice. Blocking solution composed of 3\% BSA was then introduced and incubated for $30 \mathrm{~min}$ at $37^{\circ} \mathrm{C}$ to minimise non-specific binding of antibodies. The channels were washed with PBS. The cells were incubated with the primary antibodies (anti EpCAM antibody) for $1 \mathrm{~h}$ at $37^{\circ} \mathrm{C}$. The cells were then washed gently with PBS followed by incubation with the appropriate secondary antibody for $1 \mathrm{~h}$. The cells were washed again with PBS before adding the mounting medium containing DAPI followed by sealing with a cover slip. Immunofluorescence was analysed using Leica SP5 confocal microscope. The experiments were done on two separate devices simultaneously. The diagnostic evaluation of the CTC capture assay used was estimated using the following formulae: ${ }^{61}$

$$
\begin{aligned}
& \% \text { Predictive positive }=\frac{\text { Number of true positives }}{\text { Total number of positive results }} \times 100, \\
& \% \text { Predictive negative }=\frac{\text { Number of true negative }}{\text { Total number of negative results }} \times 100, \\
& \% \text { Diagnostic efficiency }=\frac{\text { Number of true positive }+ \text { true negative results }}{\text { Number of true positive }+ \text { false positive }} \times 100 \\
& + \text { true negative }+ \text { false negative results } \\
& \% \text { Sensitivity }=\frac{\text { Number of true positive results }}{\text { Number of true positive results }+ \text { false negative results }} \times 100, \\
& \% \text { Specificity }=\frac{\text { Number of true negative results }}{\text { Number of true nega tive results }+ \text { false positive results }} \times 100,
\end{aligned}
$$

where true positives represent all the blood samples obtained from advanced stage cancer patients that showed the presence of CTCs (that were positive for CTCs) and true negatives represent all the blood samples obtained from patients with pre-cancerous lesions that did not show any CTCs (that were negative for CTCs).

\section{EpCAM ICD immunohistochemistry}

Immunohistochemistry (IHC) was performed using Novolink polymer detections system (Leica biosystems) on the deparaffinized sections of the cancer tissues as per the instructions given by the manufacturer. The tissues were rehydrated and antigen retrieval was done under steaming in a pressure cooker as per published protocol. ${ }^{62,63}$ The primary antibody, EpICD (IMG6745A, Imgenex, Novusbiologicals), was added at a dilution of 1:20 and incubated at $4{ }^{\circ} \mathrm{C}$ overnight. The tissues were DAB stained for 10 min (1:50 dilution), counter stained by hematoxylin for $30 \mathrm{~s}$, and washed twice with water. The slides were dehydrated, mounted with DPX, cover slipped, and allowed to cure overnight before observing under Olympus microscope. 


\section{Statistical analysis}

All values were expressed as mean \pm SEM obtained from at least three experiments unless otherwise stated. For the statistical evaluation of numerical data, one-way analysis of variance with Duncan's variance (SPSS 15) was used to compare the groups. $p$ values $\leq 0.05$ were considered significant, $\mathrm{p} \leq 0.01$ as highly significant, and $\mathrm{p} \leq 0.001$ as very highly significant.

\section{ACKNOWLEDGMENTS}

The authors would like to thank the Australia-India Strategic Research Fund (AISRF BF030016 and BF060042) and National Health and Medical Research Council (NHMRC APP1050286) for financial support. Financial Support was also received from Department of Biotechnology-BT/01/CEIB/11/V/16; programme support on retinoblastoma.

The manuscript was written through contributions of all authors. All authors have given approval to the final version of the manuscript.

${ }^{1}$ D. T. Miyamoto, L. V. Sequist, and R. J. Lee, "Circulating tumour cells-Monitoring treatment response in prostate cancer," Nat. Rev. Clin. Oncol. 11, 401-41 (2014).

${ }^{2}$ C. Alix-Panabières and K. Pantel, "Circulating tumor cells: Liquid biopsy of cancer," Clin. Chem. 59, 110-118 (2013).

${ }^{3}$ C. Alix-Panabières and K. Pantel, "Technologies for detection of circulating tumor cells: Facts and vision," Lab Chip 14, 57-62 (2014)

${ }^{4}$ A. Gröbe et al., "Prognostic relevance of circulating tumor cells in blood and disseminated tumor cells in bone marrow of patients with squamous cell carcinoma of the oral cavity," Clin. Cancer Res. 20, 425-433 (2014).

${ }^{5}$ W. Sheng, T. Chen, W. Tan, and Z. H. Fan, "Multivalent DNA nanospheres for enhanced capture of cancer cells in microfluidic devices," ACS Nano 7, 7067-7076 (2013).

${ }^{6} \mathrm{~S}$. Riethdorf et al., "Detection of circulating tumor cells in peripheral blood of patients with metastatic breast cancer: A validation study of the CellSearch system," Clin. Cancer Res. 13, 920-928 (2007).

${ }^{7}$ E. S. Lianidou, A. Strati, and A. Markou, "Circulating tumor cells as promising novel biomarkers in solid cancers," Crit. Rev. Clin. Lab. Sci. 51, 160-171 (2014).

${ }^{8}$ P. Li, Z. S. Stratton, M. Dao, J. Ritz, and T. J. Huang, "Probing circulating tumor cells in microfluidics," Lab Chip 13, 602-609 (2013).

${ }^{9}$ Y. Chen et al., "Rare cell isolation and analysis in microfluidics," Lab Chip 14, 626-645 (2014).

${ }^{10}$ K.-A. Hyun and H.-I. Jung, "Advances and critical concerns with the microfluidic enrichments of circulating tumor cells," Lab Chip 14, 45-56 (2014).

${ }^{11}$ A. G. Hovanessian et al., "The cell-surface-expressed nucleolin is associated with the actin cytoskeleton," Exp. Cell Res. 261, 312-328 (2000)

${ }^{12}$ M. Srivastava and H. B. Pollard, "Molecular dissection of nucleolin's role in growth and cell proliferation: New insights," FASEB J. 13, 1911-1922 (1999); PMID: 10544174

${ }^{13} \mathrm{~T}$. Watanabe et al., "Nucleolin on the cell surface as a new molecular target for gastric cancer treatment," Biol. Pharm. Bull. 33, 796-803 (2009).

${ }^{14}$ M. Herlyn, Z. Steplewski, D. Herlyn, and H. Koprowski, "Colorectal carcinoma-specific antigen: Detection by means of monoclonal antibodies,” Proc. Natl. Acad. Sci. U. S. A. 76, 1438-1442 (1979).

${ }^{15}$ M. J. Winter, I. D. Nagtegaal, J. Van Krieken, and S. V. Litvinov, "The epithelial cell adhesion molecule (Ep-CAM) as a morphoregulatory molecule is a tool in surgical pathology,” Am. J. Pathol. 163, 2139-2148 (2003).

${ }^{16} \mathrm{P}$. T. Went et al., "Frequent EpCam protein expression in human carcinomas," Human Pathol. 35, 122-128 (2004)

${ }^{17} \mathrm{P}$. Went et al.. "Frequent high-level expression of the immunotherapeutic target Ep-CAM in colon, stomach, prostate and lung cancers," Br. J. Cancer 94, 128-135 (2006).

${ }^{18}$ J. E. Visvader and G. J. Lindeman, "Cancer stem cells in solid tumours: Accumulating evidence and unresolved questions," Nat. Rev. Cancer 8, 755-768 (2008).

${ }^{19}$ B. T. F. van der Gun et al.. "EpCAM in carcinogenesis: The good, the bad or the ugly," Carcinogenesis 31, 1913-1921 (2010).

${ }^{20}$ D. Maetzel et al., "Nuclear signalling by tumour-associated antigen EpCAM," Nature Cell Biol. 11, 162-171 (2009).

${ }^{21} \mathrm{Z}$. Tang et al., "Selection of aptamers for molecular recognition and characterization of cancer cells," Anal. Chem. 79, 4900-4907 (2007).

${ }^{22}$ D. Shangguan et al., "Aptamers evolved from live cells as effective molecular probes for cancer study," Proc. Natl. Acad. Sci. 103, 11838-11843 (2006).

${ }^{23}$ M. Famulok, G. Mayer, and M. Blind, "Nucleic acid aptamers from selection in vitro to applications in vivo," Acc. Chem. Res. 33, 591-599 (2000).

${ }^{24}$ A. Z. Wang and O. C. Farokhzad, "Current progress of aptamer-based molecular imaging," J. Nucl. Med. 55, 353-356 (2014).

${ }^{25}$ Y. Xu et al., "Aptamer-based microfluidic device for enrichment, sorting, and detection of multiple cancer cells," Anal. Chem. 81, 7436-7442 (2009).

${ }^{26} \mathrm{~W}$. Sheng et al., "Aptamer-enabled efficient isolation of cancer cells from whole blood using a microfluidic device," Anal. Chem. 84, 4199-4206 (2012).

${ }^{27}$ M. A. Campbell and J. Wengel, "Locked vs. unlocked nucleic acids (LNA vs. UNA): Contrasting structures work towards common therapeutic goals," Chem. Soc. Rev. 40, 5680-5689 (2011). 
${ }^{28}$ R. N. Veedu and J. Wengel, "Locked nucleic acids: promising nucleic acid analogs for therapeutic applications," Chem. Biodivers. 7, 536-542 (2010)

${ }^{29}$ D. Shangguan, Z. Tang, P. Mallikaratchy, Z. Xiao, and W. Tan, "Optimization and modifications of aptamers selected from live cancer cell lines," ChemBioChem 8, 603-606 (2007).

${ }^{30}$ J. R. Kanwar, K. Roy, and R. K. Kanwar, "Chimeric aptamers in cancer cell-targeted drug delivery," Crit. Rev. Biochem. Mol. Biol. 46, 459-477 (2011).

${ }^{31}$ I. Cima et al., "Label-free isolation of circulating tumor cells in microfluidic devices: Current research and perspectives," Biomicrofluidics 7, 011810 (2013).

${ }^{32}$ S. Shim et al., "Antibody-independent isolation of circulating tumor cells by continuous-flow dielectrophoresis," Biomicrofluidics 7, 011807 (2013).

${ }^{33} \mathrm{H}$.-S. Moon et al., "Continual collection and re-separation of circulating tumor cells from blood using multi-stage multiorifice flow fractionation," Biomicrofluidics 7, 014105 (2013).

${ }^{34} \mathrm{~J}$. Sun et al., "Size-based hydrodynamic rare tumor cell separation in curved microfluidic channels," Biomicrofluidics 7 , $011802(2013)$

${ }^{35}$ U. Marolt, A. Cencic, M. Gorenjak, and S. Potrc, "Generating aptamers for cancer diagnosis and therapy," Clin. Exp. Pharmacol. 2, 1-8 (2012).

${ }^{36}$ Y. Takei et al., "5'-,3'-inverted thymidine-modified antisense oligodeoxynucleotide targeting midkine: Its design and application for cancer therapy,” J. Biol. Chem. 277, 23800-23806 (2002).

${ }^{37}$ C. Marquette, I. Lawrence, C. Polychronakos, and M. Lawrence, "Impedance based DNA chip for direct Tm measurement," Talanta 56, 763-768 (2002).

${ }^{38}$ See supplementary material at http://dx.doi.org/10.1063/1.4930983 for simultaneous characterization for multiple markers/proteins.

${ }^{39}$ J. A. Phillips, Y. Xu, Z. Xia, Z. H. Fan, and W. Tan, "Enrichment of cancer cells using aptamers immobilized on a microfluidic channel," Anal. Chem. 81, 1033-1039 (2008).

${ }^{40}$ A. Armstrong and S. L. Eck, "EpCAM: A new therapeutic target for an old cancer antigen," Cancer Biol. Ther. 2, 320-325 (2003).

${ }^{41}$ M. Balzar, M. J. Winter, C. J. de Boer, and S. V. Litvinov, “The biology of the 17-1A antigen (Ep-CAM),” J. Mol. Med. (Berl) 77, 699-712 (1999).

${ }^{42}$ J. R. Kanwar, R. K. Kanwar, G. Mahidhara, and C. H. A. Cheung, "Cancer targeted nanoparticles specifically induce apoptosis in cancer cells and spare normal cells," Aust. J. Chem. 65, 5-14 (2012).

${ }^{43} \mathrm{~V}$. Đapic et al., "Antiproliferative activity of G-quartet-forming oligonucleotides with backbone and sugar modifications," Biochemistry 41, 3676-3685 (2002).

${ }^{44} \mathrm{~F}$. Mongelard and P. Bouvet, "AS-1411, a guanosine-rich oligonucleotide aptamer targeting nucleolin for the potential treatment of cancer, including acute myeloid leukemia," Curr. Opin. Mol. Ther. 12, 107-114 (2010); PMID: 20140822.

${ }^{45}$ S. Shigdar et al., "RNA aptamer against a cancer stem cell marker epithelial cell adhesion molecule," Cancer Sci. 102, 991-998 (2011)

${ }^{46}$ P. J. Bates, J. B. Kahlon, S. D. Thomas, J. O. Trent, and D. M. Miller, “Antiproliferative activity of G-rich oligonucleotides correlates with protein binding," J. Biol. Chem. 274, 26369-26377 (1999).

${ }^{47}$ Q. Zhou et al., "Aptamer-containing surfaces for selective capture of CD4 expressing cells," Langmuir 28, 12544-12549 (2012).

${ }^{48} \mathrm{U}$. Dharmasiri et al., "Highly efficient capture and enumeration of low abundance prostate cancer cells using prostate-specific membrane antigen aptamers immobilized to a polymeric microfluidic device," Electrophoresis 30, 3289-3300 (2009).

${ }^{49}$ J. Zhu, T. Nguyen, R. Pei, M. Stojanovic, and Q. Lin, "Specific capture and temperature-mediated release of cells in an aptamer-based microfluidic device," Lab Chip 12, 3504-3513 (2012).

${ }^{50}$ K.-M. Song, S. Lee, and C. Ban, “Aptamers and their biological applications," Sensors 12, 612-631 (2012).

${ }^{51}$ R. R. Breaker, "Natural and engineered nucleic acids as tools to explore biology," Nature 432, 838-845 (2004)

${ }^{52} \mathrm{~W}$. Sheng et al., "Capture, release and culture of circulating tumor cells from pancreatic cancer patients using an enhanced mixing chip," Lab Chip 14, 89-98 (2014).

${ }^{53}$ K. Farin et al., "Oncogenic synergism between ErbB1, nucleolin, and mutant Ras," Cancer Res. 71, 2140-2151 (2011).

${ }^{54} \mathrm{X}$. Guo et al., "Increased level of nucleolin confers to aggressive tumor progression and poor prognosis in patients with hepatocellular carcinoma after hepatectomy," Diagn. Pathol. 9, 175 (2014).

${ }^{55} \mathrm{E}$. M. Reyes-Reyes and S. K. Akiyama, "Cell-surface nucleolin is a signal transducing P-selectin binding protein for human colon carcinoma cells," Exp. Cell Res. 314, 2212-2223 (2008).

${ }^{56} \mathrm{~V}$. Schartinger et al., "The expression of EGFR, HER2 and EpCam in head and neck squamous cell carcinomas," memo-Mag. Eur. Med. Oncol. 2, 45-50 (2009).

${ }^{57} \mathrm{H}$. J. Yoon et al., "Sensitive capture of circulating tumour cells by functionalized graphene oxide nanosheets," Nat. Nanotechnol. 8, 735-741 (2013).

${ }^{58}$ M. Schena et al., "Parallel human genome analysis: microarray-based expression monitoring of 1000 genes," Proc. Natl. Acad. Sci. 93, 10614-10619 (1996).

${ }^{59}$ D. M. Walker, G. Boey, and L. McDonald, "The pathology of oral cancer," Pathology 35, 376-383 (2003).

${ }^{60}$ S. Warnakulasuriya, "Global epidemiology of oral and oropharyngeal cancer," Oral Oncol. 45, 309-316 (2009).

${ }^{61}$ J. Kanwar and V. Vinayak, "The significance of free and immune-complexed hydatid-specific antigen (s) as an immunodiagnostic tool for human hydatidosis," J. Med. Microbiol. 37, 396-403 (1992).

${ }^{62}$ R. Miller and C. Estran, Heat-Induced Epitope Retrieval with a Pressure Cooker-Suggestions for Optimal Use (Lippincott-Raven Publ., 227 East Washington Sq, Philadelphia, PA 19106, 1995), Vol. 3, pp. 190-193.

${ }^{63}$ A. J. Norton, S. Jordan, and P. Yeomans, "Brief, high-temperature heat denaturation (pressure cooking): A simple and effective method of antigen retrieval for routinely processed tissues," J. Pathol. 173, 371-379 (1994).

${ }^{64} \mathrm{~K}$. Roy et al., "Locked nucleic acid modified bi-specific aptamer-targeted nanoparticles carrying survivin antagonist towards effective colon cancer therapy," RSC Adv. 5, 29008-29016 (2015). 